Mathematical Modelling and Analysis

Volume 20 Number 4, July 2015, 502-515

http://dx.doi.org/10.3846/13926292.2015.1070766

(c) Vilnius Gediminas Technical University, 2015
Publisher: Taylor\&Francis and VGTU

http://www.tandfonline.com/TMMA

ISSN: $1392-6292$

eISSN: $1648-3510$

\title{
Approximation of the Sections of the Set of Trajectories of the Control System Described by a Nonlinear Volterra Integral Equation
}

\author{
Anar Huseyin $^{a}$, Nesir Huseyin ${ }^{b}$ and Khalik G. Guseinov \\ ${ }^{a}$ Cumhuriyet University, Faculty of Science \\ 58140 Sivas, Turkey \\ ${ }^{b}$ Cumhuriyet University, Faculty of Education \\ 58140 Sivas, Turkey \\ ${ }^{c}$ Anadolu University, Mathematics Department \\ 26470 Eskisehir, Turkey \\ E-mail(corresp.): ahuseyin@cumhuriyet.edu.tr \\ E-mail: nhuseyin@cumhuriyet.edu.tr \\ E-mail: kguseynov@anadolu.edu.tr
}

Received February 6, 2015; revised July 3, 2015; published online July 15, 2015

\begin{abstract}
Approximation of the sections of the set of trajectories of the control system described by a nonlinear Volterra integral equation is studied. The admissible control functions are chosen from the closed ball of the space $L_{p}, p>1$, with radius $\mu$ and centered at the origin. The set of admissible control functions is replaced by the set of control functions, which includes a finite number of control functions and generates a finite number of trajectories. It is proved that the sections of the set of trajectories can be approximated by the sections of the set of trajectories, generated by a finite number of control functions.
\end{abstract}

Keywords: nonlinear Volterra integral equation, control system, integral constraint, set of trajectories, approximation method.

AMS Subject Classification: 93C23; 45D05.

\section{Introduction}

Control systems arise in various fields of theory and applications. Depending on the type of equation which describes the system's behavior, control systems can be classified as linear or nonlinear control systems, control systems described by ordinary differential equations or control systems described by partial differential equations or control systems described by integral equations and etc. Control systems can also be classified by the character of control functions, say as control systems with geometric constraints on the controls, control systems with integral constraints on the control functions and control 
systems with mixed type constraints on the control functions. In general, geometric constraints on the controls arise in cases, where control resource is not exhausted by consumption. But in the case, when control resource is exhausted by consumption, then integral constraint on the control functions is inevitable. These kinds of controls take place, if control resource is energy, fuel, finance, food and etc. (see, e.g. [5,7,16,20,24] and references therein). For example, the mathematical model of flying objects with rapidly changing mass is described as a control system with integral constraint on controls (see, $[7,16,26]$ ).

The theory of control systems with geometric constraints on the controls is a well investigated part of the control system's theory. The problems of existence of the optimal trajectories, controllability of the control systems, necessary and sufficient conditions for optimality of given processes, numerical methods for calculation of optimal trajectories and attainable sets of control systems described by an ordinary differential equation are studied in numerous papers (see, e.g. $[6,9,12,17,18,23]$ and references therein). The same problems for the systems described by a Volterra type integral equation are considered in papers $[1,2,3,21,25,29]$ (see, also the references in these papers). Various properties, including numerical methods for construction of the attainable sets of the control systems described by an ordinary differential equations with integral constraints on the controls are studied in papers $[5,7,10,11,16,19,20$, 24]. Note that integral constraint on the controls is essentially different than geometric constraint. The systems with geometric constraint on the controls can be reduced to the suitable, differential or integral inclusion (see, $[6,9,12,17]$ ). However, the same technic is not always applicable for the system with integral constraint on the controls, since integral boundedness of control functions does not guarantee the geometric boundedness of the controls, and therefore in the investigation of the control systems with integral constraints on the controls arise additional difficulties.

The mathematical models of many processes in mechanics, physics, economy, biology and etc. are described by integral equations. The Volterra integral equations are an important tool for description of mathematical models of various types of complex processes (see, e.g. [4, 8, 15, 22, 27, 28] and references therein). Some properties of the set of trajectories of the control systems described by a nonlinear Volterra integral equation with integral constraint on the controls are discussed in [13] and [14]. Precompactness of the set of trajectories of the control systems described by a nonlinear Volterra integral equation with integral constraint on the control functions is considered in [14]. In [13] an approximation of the sections of the set of trajectories of the control systems described by a nonlinear Volterra integral equation with integral constraint on the controls is investigated, where the sections of the set of trajectories is approximated by the sections of the set of trajectories generated by the compact set of control functions. In this paper, developing the result obtained in [13], the sections of the set of trajectories are approximated by the sections of the set of trajectories which consists of a finite number of trajectories.

The paper is organized as follows. In Section 2 the basic conditions are formulated which satisfy the system (Conditions 2.A, 2.B and 2.C). The known result from [13] is given which is used in the following arguments (Theorem 1). 
In Section 3, the sections of the set of trajectories are approximated by the sections of the set of trajectories, generated by the set of control functions which are piecewise constant and have mixed, i.e. integral and geometric constraints (Theorem 2).

In Section 4, an evaluation for the Hausdorff distance between the set of trajectories, generated by the piecewise constant and mixed constrained control functions and the set of trajectories, generated by the mixed constrained and piecewise constant control functions the norm of which are the node points of the given uniform mesh, is obtained (Proposition 3).

In Section 5, a new class of control functions is introduced which consists of a finite number of control functions. These control functions generate the set of trajectories consisting of a finite number of trajectories. The Hausdorff distance between the set of trajectories generated by the mixed constrained and piecewise constant control functions the norm of which are the node points of the given uniform mesh, and the set of trajectories consisting of a finite number of trajectories is evaluated (Proposition 4).

In Section 6, the main result of the paper is given. It is proved that the sections of the set of trajectories of the system can be approximated by the sections of the set of trajectories, which consists of a finite number of trajectories (Theorem 3).

The Hausdorff distance between the sets $E \subset \mathbb{R}^{n}$ and $F \subset \mathbb{R}^{n}$ is denoted by $h_{n}(E, F)$, where $\mathbb{R}^{n}$ is $n$-dimensional Euclidean space. The Hausdorff distance between the sets $G \subset C\left([a, b] ; \mathbb{R}^{n}\right)$ and $W \subset C\left([a, b] ; \mathbb{R}^{n}\right)$ is denoted by $h_{C}(G, W)$, where $C\left([a, b] ; \mathbb{R}^{n}\right)$ is the space of continuous functions $x(\cdot):[a, b] \rightarrow \mathbb{R}^{n}$ with norm $\|x(\cdot)\|_{C}=\max \{\|x(\xi)\|: \xi \in[a, b]\}$ and $\|x\|$ is the Euclidean norm of $x \in \mathbb{R}^{n}$. We set

$$
B_{n}=\left\{x \in \mathbb{R}^{n}:\|x\| \leq 1\right\}, \quad B_{C}=\left\{x(\cdot) \in C\left([a, b] ; \mathbb{R}^{n}\right):\|x(\cdot)\|_{C} \leq 1\right\} .
$$

\section{Preliminaries}

The control system described by a nonlinear Volterra integral equation

$$
x(\xi)=f(\xi, x(\xi))+\lambda \int_{a}^{\xi} K(\xi, s, x(s), u(s)) d s
$$

is considered, where $x(s) \in \mathbb{R}^{n}$ is the state vector of the system, $u(s) \in \mathbb{R}^{m}$ is the control vector, $\xi \in[a, b], \lambda \geq 0$ is a real number.

For given $p>1$ and $\mu>0$ we set

$$
U_{p, \mu}=\left\{u(\cdot) \in L_{p}\left([a, b] ; \mathbb{R}^{m}\right):\|u(\cdot)\|_{p} \leq \mu\right\},
$$

where $\|u(\cdot)\|_{p}=\left(\int_{a}^{b}\|u(s)\|^{p} d s\right)^{\frac{1}{p}}, L_{p}\left([a, b] ; \mathbb{R}^{m}\right)$ is the space of Lebesgue measurable functions $u(\cdot):[a, b] \rightarrow \mathbb{R}^{m}$ such that $\|u(\cdot)\|_{p}<\infty$. The set $U_{p, \mu} \subset$ $L_{p}\left([a, b] ; \mathbb{R}^{m}\right)$ is called the set of admissible control functions and every function $u(\cdot) \in U_{p, \mu}$ is called admissible control function.

From now on, it will be assumed that the functions $f(\cdot):[a, b] \times \mathbb{R}^{n} \rightarrow \mathbb{R}^{n}$, $K(\cdot):[a, b] \times[a, b] \times \mathbb{R}^{n} \times \mathbb{R}^{m} \rightarrow \mathbb{R}^{n}$ and number $\lambda \in[0, \infty)$ given in equation (2.1) satisfy the following conditions: 
2.A. The functions $f(\cdot):[a, b] \times \mathbb{R}^{n} \rightarrow \mathbb{R}^{n}$ and $K(\cdot):[a, b] \times[a, b] \times \mathbb{R}^{n} \times$ $\mathbb{R}^{m} \rightarrow \mathbb{R}^{n}$ are continuous;

2.B. There exist $L_{0} \in[0,1), L_{1} \geq 0, H_{1} \geq 0, L_{2} \geq 0, H_{2} \geq 0, L_{3} \geq 0$ and $H_{3} \geq 0$ such that

$$
\left\|f\left(\xi, x_{1}\right)-f\left(\xi, x_{2}\right)\right\| \leq L_{0}\left\|x_{1}-x_{2}\right\|
$$

for every $\left(\xi, x_{1}\right) \in[a, b] \times \mathbb{R}^{n},\left(\xi, x_{2}\right) \in[a, b] \times \mathbb{R}^{n}$, and

$$
\begin{aligned}
& \left\|K\left(\xi_{1}, s, x_{1}, u_{1}\right)-K\left(\xi_{2}, s, x_{2}, u_{2}\right)\right\| \leq\left[L_{1}+H_{1}\left(\left\|u_{1}\right\|+\left\|u_{2}\right\|\right)\right]\left|\xi_{1}-\xi_{2}\right| \\
& \quad+\left[L_{2}+H_{2}\left(\left\|u_{1}\right\|+\left\|u_{2}\right\|\right)\right]\left\|x_{1}-x_{2}\right\|+\left[L_{3}+H_{3}\left(\left\|x_{1}\right\|+\left\|x_{2}\right\|\right)\right]\left\|u_{1}-u_{2}\right\|
\end{aligned}
$$

for every $\left(\xi_{1}, s, x_{1}, u_{1}\right) \in[a, b] \times[a, b] \times \mathbb{R}^{n} \times \mathbb{R}^{m},\left(\xi_{2}, s, x_{2}, u_{2}\right) \in[a, b] \times[a, b] \times$ $\mathbb{R}^{n} \times \mathbb{R}^{m}$

2.C. $0 \leq \lambda\left(L_{2}(b-a)+2 H_{2}(b-a)^{\frac{p-1}{p}} \mu\right)<1-L_{0}$.

Note that if the function $K(\cdot):[a, b] \times[a, b] \times \mathbb{R}^{n} \times \mathbb{R}^{m} \rightarrow \mathbb{R}^{n}$ is Lipschitz continuous, then it satisfies the conditions $2 . \mathrm{A}$ and $2 . \mathrm{B}$.

Let us define the trajectory of the system (2.1) generated by an admissible control function $u(\cdot) \in U_{p, \mu}$. Let $u_{*}(\cdot) \in U_{p, \mu}$. A continuous function $x_{*}(\cdot)$ : $[a, b] \rightarrow \mathbb{R}^{n}$ satisfying the equation

$$
x_{*}(\xi)=f\left(\xi, x_{*}(\xi)\right)+\lambda \int_{a}^{\xi} K\left(\xi, s, x_{*}(s), u_{*}(s)\right) d s
$$

for every $\xi \in[a, b]$ is said to be a trajectory of the system (2.1) generated by the admissible control function $u_{*}(\cdot) \in U_{p, \mu}$.

The conditions 2.A-2.C guarantee that every admissible control function generates a unique trajectory of the system (2.1) (see [14]). We denote by $\mathbf{X}_{p, \mu}$ the set of all trajectories of the system (2.1) generated by all admissible control functions $u(\cdot) \in U_{p, \mu}$. The set $\mathbf{X}_{p, \mu}$ is called the set of trajectories of the system $(2.1)$.

For each fixed $\xi \in[a, b]$ we set

$$
\mathbf{X}_{p, \mu}(\xi)=\left\{x(\xi) \in \mathbb{R}^{n}: x(\cdot) \in \mathbf{X}_{p, \mu}\right\}
$$

According to [14] the set of trajectories $\mathbf{X}_{p, \mu}$ is a precompact subset of the space $C\left([a, b] ; \mathbb{R}^{n}\right)$, and hence there exists $r_{*}>0$ such that

$$
\|x(\cdot)\|_{C} \leq r_{*}
$$

for every $x(\cdot) \in \mathbf{X}_{p, \mu}$. Denote

$$
R_{*}=\frac{\lambda\left(L_{3}+2 r_{*} H_{3}\right)}{1-L_{0}} \cdot \exp \left[\frac{\lambda\left(L_{2}(b-a)+2 H_{2} \mu(b-a)^{\frac{p-1}{p}}\right)}{1-L_{0}}\right],
$$

where $r_{*}$ is defined by $(2.3)$.

The validity of the following proposition follows from conditions 2.A and 2.B. 
Proposition 1. Let $x(\cdot) \in \mathbf{X}_{p, \mu}$ and $x_{*}(\cdot) \in \mathbf{X}_{p, \mu}$ be arbitrary trajectories of the system (2.1) generated by the admissible control functions $u(\cdot) \in U_{p, \mu}$ and $u_{*}(\cdot) \in U_{p, \mu}$ respectively. Then

$$
\left\|x(\xi)-x_{*}(\xi)\right\| \leq R_{*} \int_{a}^{\xi}\left\|u(s)-u_{*}(s)\right\| d s
$$

for every $\xi \in[a, b]$.

For given $H>0, K>0$ we denote

$$
\begin{aligned}
U_{p, \mu}^{H} & =\left\{u(\cdot) \in U_{p, \mu}:\|u(\xi)\| \leq H \text { for every } \xi \in[a, b]\right\}, \\
U_{p, \mu}^{H, l i p, K} & =\left\{u(\cdot) \in U_{p, \mu}^{H}: u(\cdot):[a, b] \rightarrow \mathbb{R}^{m}\right. \text { is Lipschitz continuous and }
\end{aligned}
$$

Lipschitz's constant is not greater than $K\}$,

and let $\mathbf{X}_{p, \mu}^{H, l i p, K}$ be the set of all trajectories of the system (2.1) generated by all control functions $u(\cdot) \in U_{p, \mu}^{H, l i p, K}$. For given $\xi \in[a, b]$ we set

$$
\mathbf{X}_{p, \mu}^{H, l i p, K}(\xi)=\left\{x(\xi) \in \mathbb{R}^{n}: x(\cdot) \in \mathbf{X}_{p, \mu}^{H, l i p, K}\right\} .
$$

Theorem 1. [13] For each $\varepsilon>0$ there exist $H(\varepsilon)>0$ and $K_{*}(\varepsilon)$ $=K_{*}(\varepsilon, H(\varepsilon))>0$ such that for every $K>K_{*}(\varepsilon)$ the inequality

$$
h_{n}\left(\mathbf{X}_{p, \mu}(\xi), \mathbf{X}_{p, \mu}^{H(\varepsilon), l i p, K}(\xi)\right)<\frac{\varepsilon}{4}
$$

is verified for every $\xi \in[a, b]$, where $\mathbf{X}_{p, \mu}(\xi)$ is defined by (2.2).

\section{The Set of Trajectories Generated by the Piecewise Constant Control Functions}

Let $\Gamma=\left\{a=\xi_{0}, \xi_{1}, \ldots, \xi_{N}=b\right\}$ be a uniform partition of the closed interval $[a, b]$ and $\Delta=\xi_{i+1}-\xi_{i}, i=0,1, \ldots, N-1$. For given $H>0$ and $K>0$ we set

$$
\begin{gathered}
U_{p, \mu}^{H, \Gamma}=\left\{u(\cdot) \in U_{p, \mu}^{H}: u(\xi)=u_{i}, \xi \in\left[\xi_{i}, \xi_{i+1}\right), i=0,1, \ldots, N-1\right\}, \\
V_{p, \mu}^{H, \Gamma, K}=\left\{u(\cdot) \in U_{p, \mu}^{H}: u(\xi)=u_{i}, \xi \in\left[\xi_{i}, \xi_{i+1}\right), i=0,1, \ldots, N-1,\right. \\
\\
\left.\left\|u_{i+1}-u_{i}\right\| \leq K \Delta, i=0,1, \ldots, N-2\right\}
\end{gathered}
$$

and let $\mathbf{X}_{p, \mu}^{H, \Gamma}$ and $\mathbf{Z}_{p, \mu}^{H, \Gamma, K}$ be the set of trajectories of the system (2.1) generated by the control functions $u(\cdot) \in U_{p, \mu}^{H, \Gamma}$ and $v(\cdot) \in V_{p, \mu}^{H, \Gamma, K}$ respectively. For given $\xi \in[a, b]$ we denote

$$
\begin{aligned}
\mathbf{X}_{p, \mu}^{H, \Gamma}(\xi) & =\left\{x(\xi) \in \mathbb{R}^{n}: x(\cdot) \in \mathbf{X}_{p, \mu}^{H, \Gamma}\right\}, \\
\mathbf{Z}_{p, \mu}^{H, \Gamma, K}(\xi) & =\left\{x(\xi) \in \mathbb{R}^{n}: x(\cdot) \in \mathbf{Z}_{p, \mu}^{H, \Gamma, K}\right\} .
\end{aligned}
$$

Since $V_{p, \mu}^{H, \Gamma, K} \subset U_{p, \mu}^{H, \Gamma}$ then we obtain that

$$
\mathbf{Z}_{p, \mu}^{H, \Gamma} \subset \mathbf{X}_{p, \mu}^{H, \Gamma}
$$

for every $H>0, K=1,2, \ldots$ and uniform partition $\Gamma$ of the closed interval $[a, b]$. 
Proposition 2. For every $H>0, K>0$ and uniform partition $\Gamma=\left\{a=\xi_{0}\right.$, $\left.\xi_{1}, \ldots, \xi_{N}=b\right\}$ of the interval $[a, b]$ the inclusion

$$
\mathbf{X}_{p, \mu}^{H, l i p, K} \subset \mathbf{Z}_{p, \mu}^{H, K}+R_{*} K(b-a) \Delta \cdot B_{C}
$$

holds, and hence

$$
\mathbf{X}_{p, \mu}^{H, l i p, K}(\xi) \subset \mathbf{Z}_{p, \mu}^{H, \Gamma, K}(\xi)+R_{*} K(b-a) \Delta \cdot B_{n}
$$

for every $\xi \in[a, b]$, where $R_{*}$ is defined by $(2.4), \mathbf{X}_{p, \mu}^{H, l i p, K}(\xi)$ and $\mathbf{Z}_{p, \mu}^{H, \Gamma, K}(\xi)$ are defined by (2.5) and (3.2) respectively, $\Delta=\xi_{i+1}-\xi_{i}, i=0,1, \ldots, N-1$.

Proof. Let us choose an arbitrary $x(\cdot) \in \mathbf{X}_{p, \mu}^{H, l i p, K}$ and let $u(\cdot) \in U_{p, \mu}^{H, l i p, K}$ be the control function, generating $x(\cdot)$. We define new control function $u_{*}(\cdot)$ : $[a, b] \rightarrow \mathbb{R}^{m}$, setting

$$
\begin{aligned}
u_{*}(\xi) & =\frac{1}{\Delta} \int_{\xi_{i}}^{\xi_{i+1}} u(s) d s, \quad \xi \in\left[\xi_{i}, \xi_{i+1}\right), i=0,1, \ldots, N-1, \\
u_{*}\left(\xi_{N}\right) & =u_{*}\left(\xi_{N-1}\right) .
\end{aligned}
$$

It is obvious, that $\left\|u_{*}(\xi)\right\| \leq H$ for every $\xi \in[a, b]$. Let $\xi \in\left[\xi_{i}, \xi_{i+1}\right)$, $i=0,1, \ldots, N-1$. It follows from (3.4) and Hölder's inequality that

$$
\left\|u_{*}(\xi)\right\| \leq \frac{1}{\Delta} \int_{\xi_{i}}^{\xi_{i+1}}\|u(s)\| d s \leq \frac{1}{\Delta^{\frac{1}{p}}}\left(\int_{\xi_{i}}^{\xi_{i+1}}\|u(s)\|^{p} d s\right)^{\frac{1}{p}}
$$

and hence

$$
\int_{\xi_{i}}^{\xi_{i+1}}\left\|u_{*}(s)\right\|^{p} d s \leq \int_{\xi_{i}}^{\xi_{i+1}}\|u(s)\|^{p} d s
$$

Since the last inequality is satisfied for every $i=0,1, \ldots, N-1$ and $u(\cdot) \in$ $U_{p, \mu}^{H, l i p, K} \subset U_{p, \mu}$ then, we have

$$
\int_{a}^{b}\left\|u_{*}(s)\right\|^{p} d s \leq \int_{a}^{b}\|u(s)\|^{p} d s \leq \mu^{p}
$$

which means that $u_{*}(\cdot) \in U_{p, \mu}$. Since $\left\|u_{*}(\xi)\right\| \leq H$ for every $\xi \in[a, b]$, we conclude that $u_{*}(\cdot) \in U_{p, \mu}^{H}$.

Since $u(\cdot) \in U_{p, \mu}^{H, l i p, K}$, then $\left\|u\left(\xi_{*}\right)-u\left(\xi^{*}\right)\right\| \leq K\left|\xi_{*}-\xi^{*}\right|$ for every $\xi_{*} \in$ $[a, b]$ and $\xi^{*} \in[a, b]$. Let $i<N-1$. According to (3.4) we obtain

$$
\begin{aligned}
\left\|u_{*}\left(\xi_{i+1}\right)-u_{*}\left(\xi_{i}\right)\right\| & =\left\|\frac{1}{\Delta} \int_{\xi_{i+1}}^{\xi_{i+2}} u(s) d s-\frac{1}{\Delta} \int_{\xi_{i}}^{\xi_{i+1}} u(s) d s\right\| \\
& \leq \frac{1}{\Delta} \int_{\xi_{i}}^{\xi_{i+1}}\|u(s+\Delta)-u(s)\| d s \leq K \Delta .
\end{aligned}
$$


Since $u_{*}(\cdot) \in U_{p, \mu}^{H}$, then from (3.4) and (3.5) it follows that $u_{*}(\cdot) \in V_{p, \mu}^{H, \Gamma, K}$. Let $x_{*}(\cdot)$ be the trajectory of the system (2.1) generated by the control function $u_{*}(\cdot) \in V_{p, \mu}^{H, \Gamma, K}$. Then, $x_{*}(\cdot) \in \mathbf{Z}_{p, \mu}^{H, \Gamma, K}$ and by virtue of Proposition 1 we have

$$
\left\|x(\xi)-x_{*}(\xi)\right\| \leq R_{*} \int_{a}^{\xi}\left\|u(s)-u_{*}(s)\right\| d s
$$

for every $\xi \in[a, b]$, where $R_{*}$ is defined by (2.4).

Let us choose an arbitrary $\xi \in[a, b)$. Then there exists $i=0,1, \ldots, N-1$ such that $\xi \in\left[\xi_{i}, \xi_{i+1}\right)$. Lipschitz continuity of the control function $u(\cdot)$ and (3.4) imply that

$$
\begin{aligned}
& \left\|u(\xi)-u_{*}(\xi)\right\|=\frac{1}{\Delta}\left\|\int_{\xi_{i}}^{\xi_{i+1}} u(\xi) d s-\int_{\xi_{i}}^{\xi_{i+1}} u(s) d s\right\| \\
& \leq \frac{1}{\Delta} \int_{\xi_{i}}^{\xi_{i+1}}\|u(\xi)-u(s)\| d s \leq \frac{1}{\Delta} K \int_{\xi_{i}}^{\xi_{i+1}}|\xi-s| d s \leq K \Delta .
\end{aligned}
$$

Since $\xi \in[a, b)$ is arbitrarily chosen, we obtain from (3.6) and (3.7) that

$$
\left\|x(\xi)-x_{*}(\xi)\right\| \leq R_{*} K(b-a) \Delta
$$

for every $\xi \in[a, b]$ and consequently

$$
\left\|x(\cdot)-x_{*}(\cdot)\right\|_{C} \leq R_{*} K(b-a) \Delta,
$$

where $R_{*}$ is defined by (2.4). Thus, for arbitrarily chosen $x(\cdot) \in \mathbf{X}_{p, \mu}^{H, l i p, K}$ it is possible to define $x_{*}(\cdot) \in \mathbf{Z}_{p, \mu}^{H, \Gamma, K}$ such that the inequality (3.8) is verified. This completes the proof.

The following theorem characterizes the Hausdorff distance between the sections of sets of trajectories $\mathbf{X}_{p, \mu}$ and $\mathbf{X}_{p, \mu}^{H, \Gamma}$.

Theorem 2. For each $\varepsilon>0$ there exist $H(\varepsilon)>0$ and $\Delta_{*}(\varepsilon)>0$ such that for every uniform partition $\Gamma$ of the closed interval $[a, b]$, where $\Delta<\Delta_{*}(\varepsilon)$, the inequality

$$
h_{n}\left(\mathbf{X}_{p, \mu}(\xi), \mathbf{X}_{p, \mu}^{H(\varepsilon), \Gamma}(\xi)\right) \leq \frac{\varepsilon}{2}
$$

is verified for every $\xi \in[a, b]$. Here $\Delta$ is the diameter of the partition $\Gamma$.

Proof. According to the Theorem 1 for given $\varepsilon>0$ there exist $H(\varepsilon)>0$ and $K(\varepsilon)>0$ such that the inequality

$$
h_{n}\left(\mathbf{X}_{p, \mu}(\xi), \mathbf{X}_{p, \mu}^{H(\varepsilon), l i p, K(\varepsilon)}(\xi)\right) \leq \frac{\varepsilon}{4}
$$

is satisfied for every $\xi \in[a, b]$. By virtue of Proposition 2, for given $H(\varepsilon)>0$, $K(\varepsilon)>0$ and for every uniform partition $\Gamma=\left\{a=\xi_{0}, \xi_{1}, \ldots, \xi_{N}=b\right\}$ of the interval $[a, b]$ the inclusion

$$
\mathbf{X}_{p, \mu}^{H(\varepsilon), l i p, K(\varepsilon)}(\xi) \subset \mathbf{Z}_{p, \mu}^{H(\varepsilon), \Gamma, K(\varepsilon)}(\xi)+R_{*} K(\varepsilon)(b-a) \Delta \cdot B_{n}
$$


holds for every $\xi \in[a, b]$, where $\Delta=\xi_{i+1}-\xi_{i}, i=0,1, \ldots, N-1$.

Let $\Delta_{*}(\varepsilon)=\frac{\varepsilon}{4 R_{*} K(\varepsilon)(b-a)} \cdot$ (3.10) implies that if $\Delta<\Delta_{*}(\varepsilon)$, then

$$
\mathbf{X}_{p, \mu}^{H(\varepsilon), l i p, K(\varepsilon)}(\xi) \subset \mathbf{Z}_{p, \mu}^{H(\varepsilon), \Gamma, K(\varepsilon)}(\xi)+\frac{\varepsilon}{4} B_{n}
$$

for every $\xi \in[a, b]$.

By virtue of $(3.3)$ we have that for every uniform partition $\Gamma=\{a=$ $\left.\xi_{0}, \xi_{1}, \ldots, \xi_{N}=b\right\}$ of the interval $[a, b]$ the inclusion

$$
\mathbf{Z}_{p, \mu}^{H(\varepsilon), \Gamma, K(\varepsilon)}(\xi) \subset \mathbf{X}_{p, \mu}^{H(\varepsilon), \Gamma}(\xi)
$$

is satisfied for every $\xi \in[a, b] .(3.9),(3.11)$ and (3.12) imply that if $\Delta<\Delta_{*}(\varepsilon)$, then

$$
\mathbf{X}_{p, \mu}(\xi) \subset \mathbf{X}_{p, \mu}^{H(\varepsilon), \Gamma}(\xi)+\frac{\varepsilon}{2} B_{n}
$$

for every $\xi \in[a, b]$, where $\Delta$ is the diameter of the partition $\Gamma$. Since $\mathbf{X}_{p, \mu}^{H(\varepsilon), \Gamma}(\xi) \subset$ $\mathbf{X}_{p, \mu}(\xi)$ for every $\xi \in[a, b]$, then (3.13) completes the proof.

\section{The Set of Control Functions with Norms from Uniform Mesh}

Let $\Gamma=\left\{a=\xi_{0}, \xi_{1}, \ldots, \xi_{N}=b\right\}$ be a uniform partition of the closed interval $[a, b], H>0$ and $\Lambda=\left\{0=w_{0}, w_{1}, \ldots, w_{q}=H\right\}$ be a uniform partition of the closed interval $[0, H], \delta=w_{j+1}-w_{j}, j=0,1, \ldots, q-1$. Denote

$$
\begin{gathered}
U_{p, \mu}^{H, \Gamma}=\left\{u(\cdot) \in U_{p, \mu}^{H}: u(\xi)=u_{i}, \xi \in\left[\xi_{i}, \xi_{i+1}\right),\right. \\
\left.\left\|u_{i}\right\| \in \Lambda, i=0,1, \ldots, N-1\right\} .
\end{gathered}
$$

The set of trajectories of the system (2.1) generated by the control functions $u(\cdot) \in U_{p, \mu}^{H, \Gamma, \Lambda}$ is denoted by $\mathbf{X}_{p, \mu}^{H, \Gamma, \Lambda}$. For given $\xi \in[a, b]$ we denote

$$
\mathbf{X}_{p, \mu}^{H, \Gamma, \Lambda}(\xi)=\left\{x(\xi) \in \mathbb{R}^{n}: x(\cdot) \in \mathbf{X}_{p, \mu}^{H, \Gamma, \Lambda}\right\} .
$$

Proposition 3. For every $H>0$, uniform partition $\Gamma=\left\{a=\xi_{0}, \xi_{1}, \ldots, \xi_{N}=\right.$ b\} of the interval $[a, b]$ and uniform partition $\Lambda=\left\{0=w_{0}, w_{1}, \ldots, w_{q}=H\right\}$ of the interval $[0, H]$ the inequality

$$
h_{C}\left(\mathbf{X}_{p, \mu}^{H, \Gamma}, \mathbf{X}_{p, \mu}^{H, \Gamma, \Lambda}\right) \leq R_{*}(b-a) \delta
$$

holds, and hence

$$
h_{n}\left(\mathbf{X}_{p, \mu}^{H, \Gamma}(\xi), \mathbf{X}_{p, \mu}^{H, \Gamma}(\xi)\right) \leq R_{*}(b-a) \delta
$$

for every $\xi \in[a, b]$, where $R_{*}$ is defined by $(2.4), \mathbf{X}_{p, \mu}^{H, \Gamma}(\xi)$ and $\mathbf{X}_{p, \mu}^{H, \Gamma, \Lambda}(\xi)$ are defined by (3.1) and (4.1) respectively, $\delta=w_{j+1}-w_{j}, j=0,1, \ldots, q-1$ is the diameter of the partition $\Lambda$. 
Proof. Choose an arbitrary $x(\cdot) \in \mathbf{X}_{p, \mu}^{H, \Gamma}$ generated by the control function $u(\cdot) \in U_{p, \mu}^{H, \Gamma}$. Since $u(\cdot) \in U_{p, \mu}^{H, \Gamma}$, then $u(\xi)=u_{i}$ for every $\xi \in\left[\xi_{i}, \xi_{i+1}\right)$, $i=0,1, \ldots, N-1$, and moreover

$$
\Delta \sum_{i=0}^{N-1}\left\|u_{i}\right\|^{p} \leq \mu^{p}, \quad\left\|u_{i}\right\| \leq H \quad \text { for every } i=0,1, \ldots, N-1,
$$

where $\Delta=\xi_{i+1}-\xi_{i}, i=0,1, \ldots, N-1$, is the diameter of the partition $\Gamma$. We assume that $u(b)=u\left(\xi_{N-1}\right)$.

If $\left\|u_{i}\right\|<H(i=0,1, \ldots, N-1)$, then there exists $w_{j_{i}} \in \Lambda$ such that

$$
\left\|u_{i}\right\| \in\left[w_{j_{i}}, w_{j_{i}+1}\right) .
$$

Now, using control function $u(\cdot) \in U_{p, \mu}^{H, \Gamma}$ define a new control function $u_{*}(\cdot):[a, b] \rightarrow \mathbb{R}^{m}$, setting

$$
u_{*}(\xi)= \begin{cases}\frac{u_{i}}{\left\|u_{i}\right\|} w_{j_{i}}, & \text { if } 0<\left\|u_{i}\right\|<H, \\ u_{i}, & \text { if }\left\|u_{i}\right\|=0 \text { or }\left\|u_{i}\right\|=H,\end{cases}
$$

where $\xi \in\left[\xi_{i}, \xi_{i+1}\right), i=0,1, \ldots, N-1$, and $w_{j_{i}} \in \Lambda$ is defined by (4.3). In addition, we set that $u_{*}(b)=u_{*}\left(\xi_{N-1}\right)$. It follows from (4.3) and (4.4) that $\left\|u_{*}(\xi)\right\| \leq\|u(\xi)\|$ for every $\xi \in[a, b]$, and hence (4.2) implies that $u_{*}(\cdot) \in$ $U_{p, \mu}^{H, \Gamma, \Lambda}$. Let $x_{*}(\cdot)$ be the trajectory of the system (2.1) generated by the control function $u_{*}(\cdot)$. Then $x_{*}(\cdot) \in \mathbf{X}_{p, \mu}^{H, \Gamma, \Lambda}$ and according to the Proposition 1

$$
\left\|x(\xi)-x_{*}(\xi)\right\| \leq R_{*} \int_{a}^{\xi}\left\|u(s)-u_{*}(s)\right\| d s
$$

for every $\xi \in[a, b]$, where $R_{*}$ is defined by (2.4).

By virtue of (4.3) and (4.4) it is not difficult to verify that

$$
\left\|u(\xi)-u_{*}(\xi)\right\| \leq \delta
$$

for every $\xi \in[a, b]$, where $\delta$ is the diameter of the partition $\Lambda$. (4.5) and (4.6) yield

$$
\left\|x(\xi)-x_{*}(\xi)\right\| \leq R_{*}(b-a) \delta
$$

for every $\xi \in[a, b]$, and consequently

$$
\left\|x(\cdot)-x_{*}(\cdot)\right\|_{C} \leq R_{*}(b-a) \delta .
$$

The inequality (4.7) implies that

$$
\mathbf{X}_{p, \mu}^{H, \Gamma} \subset \mathbf{X}_{p, \mu}^{H, \Gamma}+R_{*}(b-a) \delta \cdot B_{C} .
$$

The inclusion $\mathbf{X}_{p, \mu}^{H, \Gamma, \Lambda} \subset \mathbf{X}_{p, \mu}^{H, \Gamma}$ and (4.8) imply the proof of the theorem. 


\section{Finite Number of Trajectories}

Let $\sigma>0$ be a given number and $S=\left\{u \in \mathbb{R}^{m}:\|u\|=1\right\}$. Since $S \subset \mathbb{R}^{m}$ is a compact set, then it has a finite $\sigma$-net. Let $S_{\sigma}=\left\{s_{1}, s_{2}, \ldots, s_{g}\right\}$ be a finite $\sigma$-net on $S$. We define new set of control functions, setting

$$
\begin{gathered}
U_{p, \mu}^{H, \Gamma, \Lambda, \sigma}=\left\{u(\cdot) \in U_{p, \mu}^{H, \Gamma, \Lambda}: u(\xi)=w_{j_{i}} s_{l_{i}}, \xi \in\left[\xi_{i}, \xi_{i+1}\right),\right. \\
\left.w_{j_{i}} \in \Lambda, s_{l_{i}} \in S_{\sigma}, i=0,1, \ldots, N-1\right\} .
\end{gathered}
$$

Note that the set of control functions $U_{p, \mu}^{H, \Gamma, \Lambda, \sigma}$ can be redefined as

$$
\begin{gathered}
U_{p, \mu}^{H, \Gamma, \Lambda, \sigma}=\left\{u(\cdot) \in L_{p}\left([a, b] ; \mathbb{R}^{m}\right): u(\xi)=w_{j_{i}} s_{l_{i}}, \xi \in\left[\xi_{i}, \xi_{i+1}\right), w_{j_{i}} \in \Lambda\right. \\
\left.s_{l_{i}} \in S_{\sigma}, i=0,1, \ldots, N-1, \Delta \sum_{i=0}^{N-1} w_{j_{i}}^{p} \leq \mu^{p}\right\}
\end{gathered}
$$

where $\Delta$ is the diameter of the partition $\Gamma$.

It is obvious that the set $U_{p, \mu}^{H, \Gamma, \Lambda, \sigma}$ consists of a finite number of control functions. By $\mathbf{X}_{p, \mu}^{H, \Gamma, \Lambda, \sigma}$ we denote the set of trajectories of the system (2.1) generated by the control functions $u(\cdot) \in U_{p, \mu}^{H, \Gamma, \Lambda, \sigma}$. It is obvious that the set $\mathbf{X}_{p, \mu}^{H, \Gamma, \Lambda, \sigma}$ also consists of a finite number of trajectories. For given $\xi \in[a, b]$ we set

$$
\mathbf{X}_{p, \mu}^{H, \Gamma, \Lambda, \sigma}(\xi)=\left\{x(\xi) \in \mathbb{R}^{n}: x(\cdot) \in \mathbf{X}_{p, \mu}^{H, \Gamma, \Lambda, \sigma}\right\} .
$$

Proposition 4. For every $H>0$, uniform partition $\Gamma=\left\{a=\xi_{0}, \xi_{1}, \ldots\right.$, $\left.\xi_{N}=b\right\}$ of the interval $[a, b]$, uniform partition $\Lambda=\left\{0=w_{0}, w_{1}, \ldots, w_{q}=H\right\}$ of the interval $[0, H]$ and $\sigma$-net $S_{\sigma}$, the inequality

$$
h_{C}\left(\mathbf{X}_{p, \mu}^{H, \Gamma, \Lambda}, \mathbf{X}_{p, \mu}^{H, \Gamma, \Lambda, \sigma}\right) \leq R_{*} H(b-a) \sigma
$$

holds, and hence

$$
h_{n}\left(\mathbf{X}_{p, \mu}^{H, \Gamma, \Lambda}(\xi), \mathbf{X}_{p, \mu}^{H, \Gamma, \Lambda, \sigma}(\xi)\right) \leq R_{*} H(b-a) \sigma
$$

for every $\xi \in[a, b]$, where $R_{*}$ is defined by $(2.4), \mathbf{X}_{p, \mu}^{H, \Gamma, \Lambda}(\xi)$ and $\mathbf{X}_{p, \mu}^{H, \Gamma, \Lambda, \sigma}(\xi)$ are defined by (4.1) and (5.1) respectively.

Proof. Let us choose an arbitrary $x(\cdot) \in \mathbf{X}_{p, \mu}^{H, \Gamma, \Lambda}$ generated by the control function $u(\cdot) \in U_{p, \mu}^{H, \Gamma, \Lambda}$. Since $u(\cdot) \in U_{p, \mu}^{H, \Gamma, \Lambda}$, then $\|u(\xi)\|=w_{j_{i}}$ for every $\xi \in$ $\left[\xi_{i}, \xi_{i+1}\right), i=0,1, \ldots, N-1, w_{j_{i}} \in \Lambda, \Delta \cdot \sum_{i=0}^{N-1} w_{j_{i}}^{p} \leq \mu^{p}$, where $\Delta=\xi_{i+1}-\xi_{i}$, $i=0,1, \ldots, N-1$, is the diameter of the partition $\Gamma$.

Since $\|u(\xi)\|=w_{j_{i}}$ for every $\xi \in\left[\xi_{i}, \xi_{i+1}\right), i=0,1, \ldots, N-1$, then there exist $b_{i} \in S, i=0,1, \ldots, N-1$, such that

$$
u(\xi)=w_{j_{i}} b_{i} \quad \text { for every } \xi \in\left[\xi_{i}, \xi_{i+1}\right), i=0,1, \ldots, N-1 .
$$

Since $b_{i} \in S, i=0,1, \ldots, N-1$, then there exist $s_{l_{i}} \in S_{\sigma}, i=0,1, \ldots, N-1$, such that

$$
\left\|b_{i}-s_{l_{i}}\right\| \leq \sigma, \quad i=0,1, \ldots, N-1
$$


Define new control function $u_{*}(\cdot):[a, b] \rightarrow \mathbb{R}^{m}$ setting

$$
u_{*}(\xi)=w_{j_{i}} s_{l_{i}} \quad \text { for every } \xi \in\left[\xi_{i}, \xi_{i+1}\right), i=0,1, \ldots, N-1 \text {. }
$$

It is not difficult to verify that $u_{*}(\cdot) \in U_{p, \mu}^{H, \Gamma, \Lambda, \sigma}$. Let $x_{*}(\cdot)$ be the trajectory, generated by the control function $u_{*}(\cdot)$. Then $x_{*}(\cdot) \in \mathbf{X}_{p, \mu}^{H, \Gamma, \Lambda, \sigma}$ and according to the Proposition 1 we have

$$
\left\|x(\xi)-x_{*}(\xi)\right\| \leq R_{*} \int_{a}^{\xi}\left\|u(s)-u_{*}(s)\right\| d s
$$

for every $\xi \in[a, b]$, where $R_{*}$ is defined by (2.4). (5.2), (5.3) and (5.4) yield that $\left\|u(\xi)-u_{*}(\xi)\right\| \leq H \sigma$ for every $\xi \in[a, b)$. From this inequality and (5.5) we obtain

$$
\left\|x(\xi)-x_{*}(\xi)\right\| \leq R_{*} H(b-a) \sigma
$$

for every $\xi \in[a, b]$, and hence

$$
\left\|x(\cdot)-x_{*}(\cdot)\right\|_{C} \leq R_{*} H(b-a) \sigma .
$$

(5.6) implies that

$$
\mathbf{X}_{p, \mu}^{H, \Gamma, \Lambda} \subset \mathbf{X}_{p, \mu}^{H, \Lambda, \sigma}+R_{*}(b-a) H \sigma \cdot B_{C} .
$$
rem.

Since $\mathbf{X}_{p, \mu}^{H, \Gamma, \Lambda, \sigma} \subset \mathbf{X}_{p, \mu}^{H, \Gamma, \Lambda}$, then from (5.7) we obtain the proof of the theo-

\section{Approximation}

The following theorem asserts that the sections of the set of trajectories $\mathbf{X}_{p, \mu}(\xi)$, $\xi \in[a, b]$, can be approximated by the sets $\mathbf{X}_{p, \mu}^{H, \Gamma, \Lambda, \sigma}(\xi), \xi \in[a, b]$, which consists of a finite number of points.

Theorem 3. For each $\varepsilon>0$ there exist $H(\varepsilon)>0, \Delta_{*}(\varepsilon)>0, \delta_{*}(\varepsilon)>0$ and $\sigma_{*}(\varepsilon)>0$ such that for every uniform partition $\Gamma$ of the closed interval $[a, b]$, uniform partition $\Lambda$ of the closed interval $[0, H(\varepsilon)]$ and $\sigma$-net $S_{\sigma}$, where $\Delta<\Delta_{*}(\varepsilon), \delta<\delta_{*}(\varepsilon), \sigma<\sigma_{*}(\varepsilon)$, the inequality

$$
h_{n}\left(\mathbf{X}_{p, \mu}(\xi), \mathbf{X}_{p, \mu}^{H(\varepsilon), \Gamma, \Lambda, \sigma}(\xi)\right) \leq \varepsilon
$$

is satisfied for every $\xi \in[a, b]$. Here $\Delta$ is the diameter of the partition $\Gamma, \delta$ is the diameter of the partition $\Lambda$.

Proof. According to the Theorem 2, for given $\varepsilon>0$ there exist $H(\varepsilon)>0$ and $\Delta_{*}(\varepsilon)>0$ such that for every uniform partition $\Gamma$ of the closed interval $[a, b]$, where $\Delta<\Delta_{*}(\varepsilon)$, the inequality

$$
h_{n}\left(\mathbf{X}_{p, \mu}(\xi), \mathbf{X}_{p, \mu}^{H(\varepsilon), \Gamma}(\xi)\right) \leq \frac{\varepsilon}{2}
$$


is verified for every $\xi \in[a, b]$, where $\Delta$ is the diameter of the partition $\Gamma$.

Now, by virtue of Proposition 3, for given $H(\varepsilon)>0$, for every uniform partition $\Gamma$ of the interval $[a, b]$ such that $\Delta<\Delta_{*}(\varepsilon)$, and for every uniform partition $\Lambda$ of the interval $[0, H(\varepsilon)]$ the inequality

$$
h_{n}\left(\mathbf{X}_{p, \mu}^{H(\varepsilon), \Gamma}(\xi), \mathbf{X}_{p, \mu}^{H(\varepsilon), \Gamma, \Lambda}(\xi)\right) \leq R_{*}(b-a) \delta
$$

holds for every $\xi \in[a, b]$, where $R_{*}$ is defined by $(2.4), \mathbf{X}_{p, \mu}^{H, \Gamma}(\xi)$ and $\mathbf{X}_{p, \mu}^{H, \Gamma, \Lambda}(\xi)$ are defined by (3.1) and (4.1) respectively, $\Delta$ is the diameter of the partition $\Gamma, \delta$ is the diameter of the partition $\Lambda$. If $\delta<\delta_{*}(\varepsilon)=\frac{\varepsilon}{4 R_{*}(b-a)}$, then it follows from (6.2) that for given $H(\varepsilon)>0$, for every uniform partition $\Gamma$ of the interval $[a, b]$ such that $\Delta<\Delta_{*}(\varepsilon)$, and for every uniform partition $\Lambda$ of the interval $[0, H(\varepsilon)]$ such that $\delta<\delta_{*}(\varepsilon)$, the inequality

$$
h_{n}\left(\mathbf{X}_{p, \mu}^{H(\varepsilon), \Gamma}(\xi), \mathbf{X}_{p, \mu}^{H(\varepsilon), \Gamma, \Lambda}(\xi)\right) \leq \frac{\varepsilon}{4}
$$

is satisfied for every $\xi \in[a, b]$.

From Proposition 4 we obtain that, for given $H(\varepsilon)>0$, for every uniform partition $\Gamma$ of the interval $[a, b]$ such that $\Delta<\Delta_{*}(\varepsilon)$, for every uniform partition $\Lambda$ of the interval $[0, H]$ such that $\delta<\delta_{*}(\varepsilon)$, and for every $\sigma$-net $S_{\sigma}$ the inequality

$$
h_{n}\left(\mathbf{X}_{p, \mu}^{H(\varepsilon), \Gamma, \Lambda}(\xi), \mathbf{X}_{p, \mu}^{H(\varepsilon), \Gamma, \Lambda, \sigma}(\xi)\right) \leq R_{*} H(\varepsilon)(b-a) \sigma
$$

is verified for every $\xi \in[a, b]$, where $R_{*}$ is defined by $(2.4), \mathbf{X}_{p, \mu}^{H, \Gamma, \Lambda}(\xi)$ and $\mathbf{X}_{p, \mu}^{H, \Gamma, \Lambda, \sigma}(\xi)$ are defined by (4.1) and (5.1) respectively, $\Delta$ is the diameter of the partition $\Gamma, \delta$ is the diameter of the partition $\Lambda$. If $\sigma<\sigma_{*}(\varepsilon)=\frac{\varepsilon}{4 R_{*} H(\varepsilon)(b-a)}$, then it follows from (6.4) that for given $H(\varepsilon)>0$, for every uniform partition $\Gamma$ of the interval $[a, b]$ such that $\Delta<\Delta_{*}(\varepsilon)$, for every uniform partition $\Lambda$ of the interval $[0, H(\varepsilon)]$ such that $\delta<\delta_{*}(\varepsilon)$, and for every $\sigma$-net $S_{\sigma}$ such that $\sigma<\sigma_{*}(\varepsilon)$ the inequality

$$
h_{n}\left(\mathbf{X}_{p, \mu}^{H(\varepsilon), \Gamma, \Lambda}(\xi), \mathbf{X}_{p, \mu}^{H(\varepsilon), \Gamma, \Lambda, \sigma}(\xi)\right) \leq \frac{\varepsilon}{4}
$$

is held for every $\xi \in[a, b]$.

(6.1), (6.3) and (6.5) imply that for given $\varepsilon>0$ there exist $H(\varepsilon)>0$, $\Delta_{*}(\varepsilon)>0, \delta_{*}(\varepsilon)>0$ and $\sigma_{*}(\varepsilon)>0$ such that for every uniform partition $\Gamma$ of the closed interval $[a, b]$, for every uniform partition $\Lambda$ of the closed interval $[0, H(\varepsilon)]$, for every $\sigma$-net $S_{\sigma}$, where $\Delta<\Delta_{*}(\varepsilon), \delta<\delta_{*}(\varepsilon), \sigma<\sigma_{*}(\varepsilon)$, the inequality

$$
h_{n}\left(\mathbf{X}_{p, \mu}(\xi), \mathbf{X}_{p, \mu}^{H(\varepsilon), \Gamma, \Lambda, \sigma}(\xi)\right) \leq \frac{\varepsilon}{2}+\frac{\varepsilon}{4}+\frac{\varepsilon}{4}=\varepsilon
$$

is satisfied for every $\xi \in[a, b]$.

\section{Conclusion}

In the paper an approximation method for construction of the sections of the set of trajectories of the control system described by a nonlinear Volterra type 
integral equation with integral constraint on the control functions is given. The convergence of the approximations is proved. Using the algorithm presented in [11], which allows to align the finite number of piecewise constant control functions from the set of controls $U_{p, \mu}^{H(\varepsilon), \Gamma, \Lambda, \sigma}$, and an arbitrary numerical method for calculation of the solution of nonlinear Volterra type integral equation, it is possible to carry out calculation of the section $\mathbf{X}_{p, \mu}^{H(\varepsilon), \Gamma, \Lambda, \sigma}(\xi)$. The approximate construction of the sections permits the solution of various types of optimal control problems arising in different fields of theory and applications.

\section{References}

[1] T.S. Angell. On the optimal control of systems governed by nonlinear Volterra equations. J. Optim. Theory Appl., 19:29-45, 1976. http://dx.doi.org/10.1007/BF00934050.

[2] V.L. Bakke. A maximum principle for an optimal control problem with integral constraints. J. Optim. Theory Appl., 13:32-55, 1974. http://dx.doi.org/10.1007/BF00935608.

[3] S.A. Belbas. A new method for optimal control of Volterra integral equations. Appl. Math. Comput., 189:1902-1915, 2007. http://dx.doi.org/10.1016/j.amc.2006.12.077.

[4] F. Brauer. On a nonlinear integral equation for population growth problems. SIAM J. Math. Anal., 6:312-317, 1975. http://dx.doi.org/10.1137/0506031.

[5] A.G. Chentsov. Approximative realization of integral constraints and generalized constructions in the class of vector finitely additive measures. Proc. Steklov Inst. Math., Suppl. 2:S10-S60, 2002.

[6] F.H. Clarke, Yu.S. Ledyayev, R.J. Stern and P.R. Wolenski. Nonsmooth Analysis and Control Theory. Springer, New York, 1998.

[7] R. Conti. Problemi di Controllo e di Controllo Ottimale. UTET, Torino, 1974.

[8] C. Corduneanu. Integral Equations and Applications. Cambridge University Press, Cambridge, 1991.

[9] K. Deimling. Multivalued Differential Equations. Walter De Gruyter, Berlin, 1992.

[10] K.G. Guseinov and A.S. Nazlipinar. An algorithm for approximate calculation of the attainable sets of the nonlinear control systems with integral constraint on controls. Comput. Math. Appl., 62:1887-1895, 2011. http://dx.doi.org/10.1016/j.camwa.2011.06.032.

[11] K.G. Guseinov, O. Ozer, E. Akyar and V.N. Ushakov. The approximation of reachable sets of control systems with integral constraint on controls. NoDEA Nonlinear Differential Equations Appl., 14:57-73, 2007. http://dx.doi.org/10.1007/s00030-006-4036-6.

[12] Kh.G. Guseinov, A.N. Moiseyev and V.N. Ushakov. On the approximation of reachable domains of control systems. J. Appl. Math. Mech., 62:169-175, 1998. http://dx.doi.org/10.1016/S0021-8928(98)00022-7.

[13] A. Huseyin. On the approximation of the set of trajectories of control system described by a Volterra integral equation. Nonlinear Anal. Model. Control, 19:199-208, 2014. 
[14] A. Huseyin and N. Huseyin. Precompactness of the set of trajectories of the controllable system described by a nonlinear Volterra integral equation. Math. Model. Anal., 17:686-695, 2012. http://dx.doi.org/10.3846/13926292.2012.736088.

[15] M.A. Krasnoselskii and S.G. Krein. On the principle of averaging in nonlinear mechanics. Uspekhi Mat. Nauk, 10:147-153, 1955. (in Russian)

[16] N.N. Krasovskii. Theory of Control of Motion: Linear Systems. Nauka, Moscow, 1968. (in Russian)

[17] N.N. Krasovskii and A.I. Subbotin. Game - Theoretical Control Problems. Springer-Verlag, New York, 1988.

[18] A.B. Kurzhanski and L. Valyi. Ellipsoidal Calculus for Estimation and Control. Birkhauser, Boston, 1996.

[19] H.W. Lou. On the attainable sets of control systems with $p$-integrable controls. J. Optim. Theory Appl., 123:123-147, 2004. http://dx.doi.org/10.1023/B:JOTA.0000043994.99474.ff.

[20] M. Motta and C. Sartori. Minimum time with bounded energy, minimum energy with bounded time. SIAM J. Control Optim., 42:789-809, 2003. http://dx.doi.org/10.1137/S0363012902385284.

[21] L.W. Neustadt and J. Warga. Comments on the paper Optimal control of processes described by integral equations. I by V.R. Vinokurov. SIAM J. Control Optim., 8:572, 1970. http://dx.doi.org/10.1137/0308041.

[22] A.D. Polyanin and A.V. Manzhirov. Handbook of Integral Equations. CRC Press, Boca Raton, FL, 1998.

[23] L.S. Pontryagin, V.G. Boltyanskii, R.V. Gamkrelidze and E.F. Mishchenko. The Mathematical Theory of Optimal Processes. John Wiley \& Sons, New York, 1962 .

[24] A.I. Subbotin and N.N. Subbotina. Alternative for the encounter-evasion differential game with constraints on the momenta of the players controls. J. Appl. Math. Mech., 39:376-385, 1975.

http://dx.doi.org/10.1016/0021-8928(75)90002-7.

[25] E. Tohidi and O.R.N. Samadi. Optimal control of nonlinear Volterra integral equations via Legendre polynomials. IMA J. Math. Control Inform., 30:67-83, 2013. http://dx.doi.org/10.1093/imamci/dns014.

[26] V.I. Ukhobotov. One Dimensional Projection Method in Linear Differential Games with Integral Constraints. Chelyabinsk State University Press, Chelyabinsk, 2005. (in Russian)

[27] G. Vainikko and I. Zolk. Fast spline quasicollocation solvers of integral equations. Math. Model. Anal., 12:515-538, 2007. http://dx.doi.org/10.3846/1392-6292.2007.12.515-538.

[28] M. Vath. Volterra and Integral Equations of Vector Functions. M. Dekker, New York, 2000.

[29] V.R. Vinokurov. Optimal control of processes described by integral equations. Parts I, II, III. SIAM J. Control Optim., 7:324-355, 1969.

http://dx.doi.org/10.1137/0307022. 